\title{
Experimental studies into mechanisms of cardiac arrest
}

\author{
D. C. RUSSELL \\ Cardiovascular Research Unit, University of Edinburgh, Edinburgh
}

\section{SUMMARY}

Experimental studies have revealed that a wide variety of different pathophysiological mechanisms may induce ventricular fibrillation (VF) and cardiac arrest during acute myocardial ischaemia or infarction. Distinct phases of enhanced vulnerability (the amount of current required to stimulate ectopic activity in the heart following application of an extra stimulus) to VF follow coronary occlusion and correspond to 'pre-hospital', 'in-hospital' and 'out-of-hospital' periods of arrhythmogenesis. Electrophysiological evidence suggests very early (phase 1a) VF results from multiple reentrant excitation within the ischaemic zone. Slowed and fragmented conduction and inhomogeneities in refractoriness rapidly develop which mapping studies show to occur in association with development of spatial inhomogeneities in residual blood flow distribution and metabolism. Onset of VF may be triggered by adrenergic mechanisms or influenced by peripheral metabolic responses. Automatic mechanisms (spontaneous pacemaker activity) may induce later VF or VF on reperfusion or trigger re-entry. Findings indicate no single therapeutic approach to be likely to protect against all forms of cardiac arrest.

\section{INTRODUCTION}

Cardiac arrest in the context of acute myocardial ischaemia or infarction results in general from the sudden genesis of ventricular fibrillation (VF). Although asystolic arrest may occur it is less common and is often preceded by VF.

Clinical, epidemiological and post-mortem evidence has shed little light on the underlying pathophysiological mechanisms of this phenomenon. Between $80-90 \%$ of sudden cardiac arrests are associated with severe obstructive coronary artery disease in at least one and often two or three major vessels (Kuller, 1966).

Correspondence: $\operatorname{Dr}$ D. C. Russell, Cardiovascular Research Unit, Hugh Robson Building, George Square, Edinburgh EH8 $9 X F$ 
In over a third of cases, however, no apparent clinical precipitating event is detected $\stackrel{\mathbb{\Phi}}{3}^{\mathrm{m}}$ Furthermore, of arrests associated with acute myocardial infarction the majority occuro early, within the first $1 \frac{1}{2} \mathrm{~h}$ following onset of symptoms either out-of-hospital or in the accident and emergency department and before development of definite cellular. necrosis (Armstrong et al., 1972).

Clearly, examination of underlying pathophysiological events is difficult if not impossible in man and recourse must be had to suitable experimental models. Using this approach, considerable advances have been made recently regarding mechanisms?

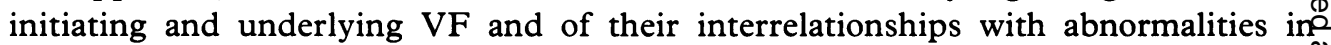
cardiac metabolism, regional myocardial blood flow and neurogenic influences on the heart. It has become clear that VF may result from a wide variety of differen $\vec{p}$ mechanisms dependent upon the duration and severity of myocardial ischaemia. Some $\vec{\omega}$ of these recent findings will be described in greater detail.

\section{The phasic nature of vulnerability to VF}

Clinical and experimental studies show phases of enhanced ventricular vulnerability to fibrillation following coronary occlusion, each with quite different mechanisms of pathogenesis and susceptibility to pharmacological intervention (Bigger et al., 1977) Vulnerability is the amount of current required to stimulate ectopic activity in the hear $\xi_{0}$ following application of an extra stimulus. Clinically a so-called 'pre-hospital' phase early arrythmogenesis occurs within the first 2 or $3 \mathrm{~h}$ after onset of symptoms myocardial infarction, with a very high incidence of VF. The 'sudden cardiac dea phenomenon may be included in this group. From 4-6h onwards a later or so-called 'in-hospital' or 'CCU' phase of arrhythmogenesis occurs with much lower incidence of VF and higher incidence of ventricular tachycardia and idio-ventricular rhythms Finally between 3 days and up to a month or more after infarction a less distinct period? of later or 'chronic' or 'out-of-hospital' arrhythmia results.

Experimental studies suggest that this picture is even more complex. In a variety off animal preparations at least two distinct periods of early arrhythmias occur within the first $30 \mathrm{~min}$ of acute coronary occlusion (Kaplinsky et al., 1979). These have beent designated phases $1 \mathrm{a}$ and $1 \mathrm{~b}$ respectively, either of which may initiate VF. Later between 4 and $6 \mathrm{~h}$ after the onset of ischaemia a period of enhanced automatic of spontaneous pacemaker activity ensues sometimes initiating VF. This is thought to reflect delayed ischaemic injury to the more hypoxia-resistant specialised conducting tissue within the heart. Re-entry, characterised by formation of electrical circus movements within the myocardium may occur also as during earlier arrhythmias, and a multiplicity of metabolic and neurogenic factors may modulate the responses. From 30 or 4 days onward, with organisation of the infarct and preservation of areas of 'chronicto ischaemic injury yet further types of arrhythmias result in association with formation of stable anatomical re-entry circuits into and within the ischaemic zone. Some of thes arrhythmias appear to be catecholamine mediated whereas others are associated witke fixed delayed depolarisation conducive to re-entry.

In addition, and dependent upon the duration of preceding ischaemia, VF may result from sudden reperfusion of an occluded coronary vessel, the clinical parallel beingo 
perhaps sudden release of coronary vasospasm or intravascular thrombolysis. Several workers believe this mechanism to be of more importance in relation to the sudden cardiac death phenomenon than ischaemia related VF per se. These arrhythmias have been further subdivided into immediate and delayed types of reperfusion VF each with differing mechanisms of pathogenesis (Kaplinsky et al., 1980).

\section{Electrophysiological mechanisms of VF}

Electrophysiological mechanisms of both initiation and perpetuation of VF are still incompletely understood. Fibrillation itself is thought to result from incoordinated electrical circus movements of excitation at multiple sites throughout the myocardium with continuous 're-entrant' excitation from one area to another (Surawicz, 1971). Opinions differ, however, as to the presence of an alternative possible mechanism of spontaneous genesis of automatic foci within the myocardium which themselves might initiate local re-entrant circuits.

The vivid description of VF given by Garry in 1924 has not to date been bettered.

'... the general process of fibrillating contraction of heart muscle may be described as an inco-ordinate, disorderly and extremely bizarre contractile process in which normal systole and diastole no longer occur ... The surface of the fibrillating chamber shows areas of fine twitchings, of fluttering and tremulous movements, combined with coarser undulating waves of muscle contraction which progress slowly through the muscle mass moving now in one direction, now in another, being continually blocked in their progress by interference with other waves'.

These patterns of mechanical activity directly result from patterns of propagation of excitation within the myocardium. These in turn are dependent upon the multiple factors determining the generation of abnormal impulses and the manner of their propagation.

Experimental studies suggest abnormal impulse generation may result from a variety of situations which include re-entrant excitation within the myocardium or specialized conducting tissue or between the two (Wit et al., 1974), enhancement of automatic or pacemaker activity, focal excitation triggered by flow of local currents of injury or by mechanical factors (Janse et al., 1980); or after-depolarisations triggering local activity. Maintenance of VF however is dependent upon continued propagation of such abnormal impulses. Isolated tissue studies indicate this relates to factors such as the state of depolarization of the cell membrane, the behaviour of specific ionic channels within the cell membrane and the ability of the impulse to pass from cell to cell (Cranefield, 1975). It seems probable that under appropriate conditions a wide variety of different mechanisms, either singly or in combination, could initiate VF in the same heart.

The dominant mechanism of early arrhythmogenesis appears to be re-entry possibly initiated by excessive flow of a current of injury across the boundary between normal and ischaemic tissue. By contrast, the later 'CCU' type VF and certain reperfusion arrhythmias are thought to be largely automatic in origin, whereas even later 'chronic' arrhythmias may arise by a combination of both re-entrant and automatic activity (Wit, 


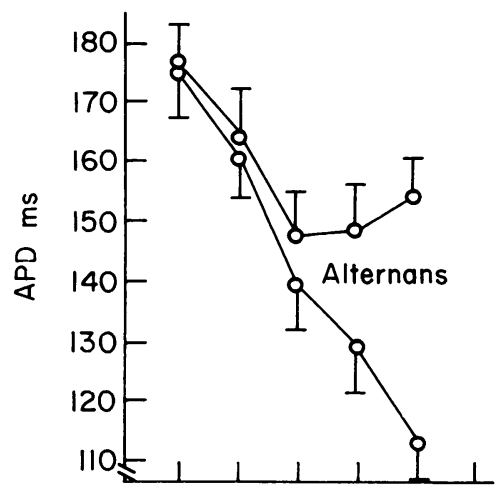

Fig. 1 Changes in action potential duration (APD), endocardial epicardial conduction delay $\frac{\mathbb{Q}}{0}$ (CD) and frequency of occurrence of ischaemic 3 zone conduction block and alternating phenomeri during 20 periods of coronary occlusions resulting. in phase 1a VF in the dog. Maximal changes occor immediately preceding VF consistent with the concept that re-entrant circuits are generated within the ischaemic zone of myocardium. (Reproduced by permission).
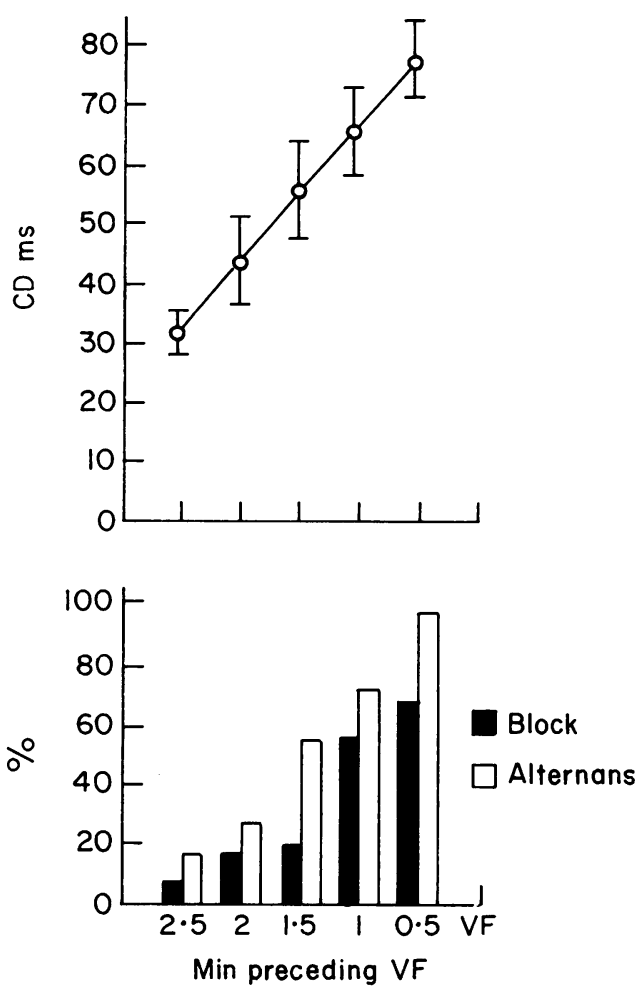

1982). Studies in our laboratory have been directed primarily towards elucidating mechanisms of genesis of the very early phase la period of VF. This will be discussed ir some greater detail.

Both macro and micro re-entry circuits have been demonstrated at this time in the pig and the dog (Janse et al., 1980) and continuous electrical activity during the course of ventricular tachycardias preceding VF shown. Such abnormal patterns of impuls propagation, however, must be determined by the distribution of electrophysiological abnormalities within the ischaemic zone. Abnormalities known at least experimentally్ㅗㅇ to predispose to re-entrant activity include slowed conduction (El-Sherif et al., 1975) development of unidirectional conduction blocks (Lazzara et al., 1978) and regiona $\frac{P}{\mathrm{D}}$ 
inhomogeneities in conduction or refractoriness to propagation of an impulse following extra-stimuli (Allesie et al., 1976). Studies were designed therefore in our laboratory to determine the presence or absence of these phenomena with relation to VF using the experimental model of acute coronary ligation in the open chest anaesthetised dog.

A 'floating' micro-electrode technique was used to obtain in vivo recordings of intracellular cardiac action potentials (Fig. 1) within the first few minutes of ischaemia and VF was found to occur at the time of maximum incidence of conduction delay, conduction block and electrical alternans (probably reflecting local 2:1 conduction block) and of shortening of action potential duration (Russell et al., 1979). Furthermore, the morphology of potentials recorded immediately preceding fibrillation was very similar to that of slowly conducting so-called 'slow response' potentials which may be recorded in vitro under conditions of partial depolarisation of the cell membrane. These slow potentials exhibit conduction properties exactly comparable with those required for re-entry. They are thought to be mediated by slow inward current carried largely by calcium ions (Cranefield, 1975).

Observations of regional variability in excitability thresholds and refractoriness immediately preceding VF provide further evidence for re-entrant mechanisms. Gross disparities in refractoriness may occur between central and border regions of the acutely ischaemic zone immediately preceding VF. Spatial inhomogeneity in recovery of excitability is generated therefore across the ischaemic zone. This is sufficient to allow the asynchrony of activation necessary for induction of VF (Russell et al., 1978).

More recently surface mapping techniques have been used to demonstrate the actual patterns of conduction within the acutely ischaemic myocardium immediately preceding VF (Russell et al., 1983). Using an 80 point multi-electrode grid sutured over a $4 \times 5 \mathrm{~cm}$ area of left ventricle overlying the ischaemic area (Fig. 2) and analysis of simultaneously derived electrograms (obtained using an electronic multiplexdemultiplex system) at successive points in time preceding induction of VF, it has been possible to construct isochronal maps of epicardial activation patterns within the ischaemic zone. Computer graphics aid display of the complex changes observed.

A representative computer graphic plot of abnormal surface epicardial delays during

Left atrial cannula

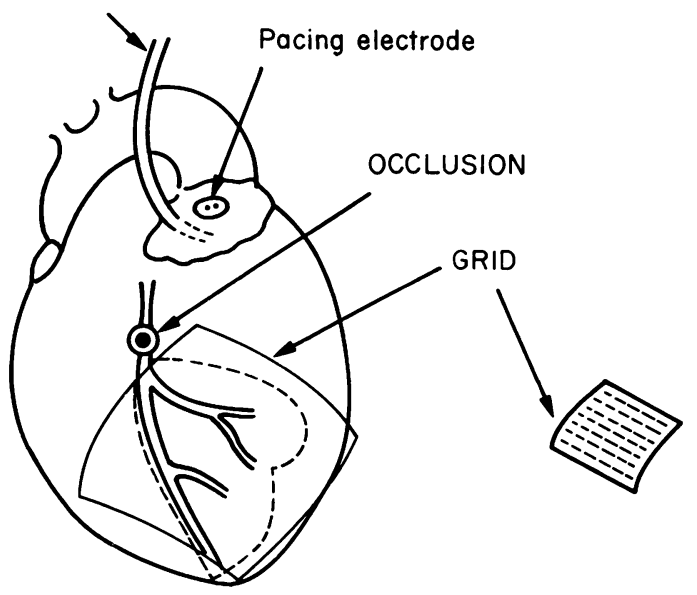

Fig. 2 Experimental design used in mapping studies. Early ventricular arrhythmias and VF are induced following proximal left anterior descending coronary artery occlusion in open chest anaesthetised dogs. Mapping is performed of regional flow and metabolism within a $4 \times 5 \mathrm{~cm}$ area underlying an 80-point flexible multielectrode grid. Computer graphics shown in Fig. 3 refer to this area. 
(a) Epicardial delay
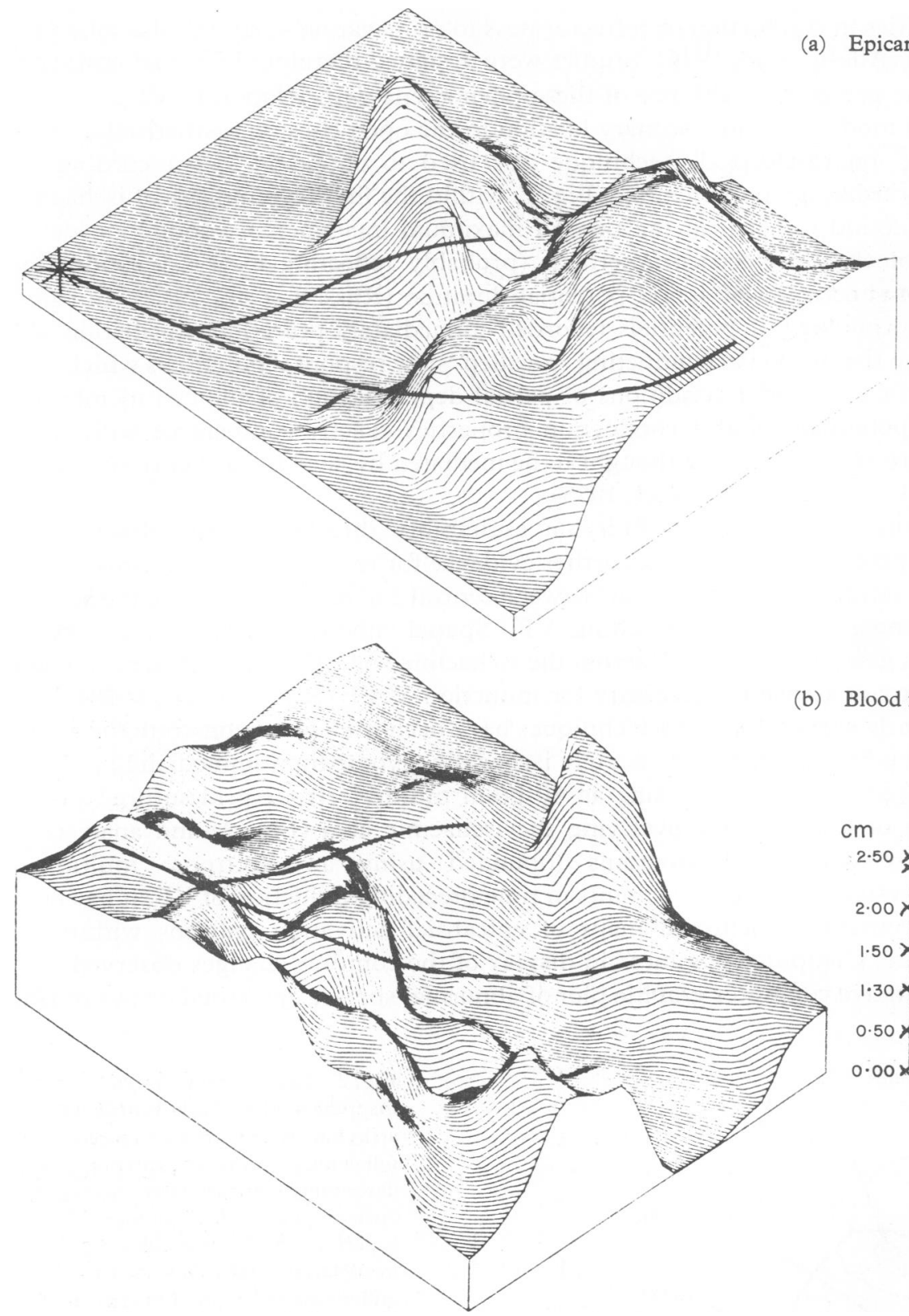

(b) Blood flow
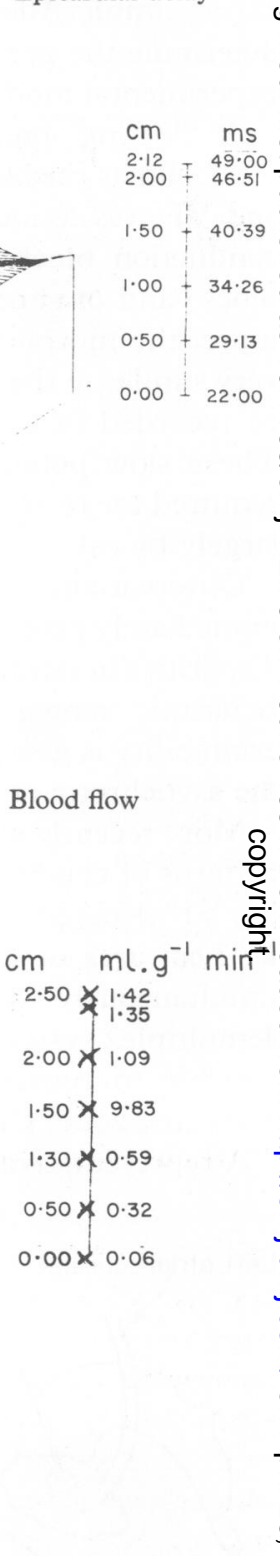

the phase 1a period of enhanced vulnerability to VF is shown in Fig. 3. The position of the left anterior descending coronary artery is shown. Peaks on the maps refer to areas of grossly abnormal conduction delays. The spatial heterogeneity of conductior abnormalities is obvious and is highly conducive to re-entrant activity. Further studieș? suggest that this heterogenous appearance itself may derive from a temporal dispersion within the myocardium of the rate of development or amelioration of conduction abnormalities. Similar features are not observed during the later $1 \mathrm{~b}$ phase which mas arise by different mechanisms. 


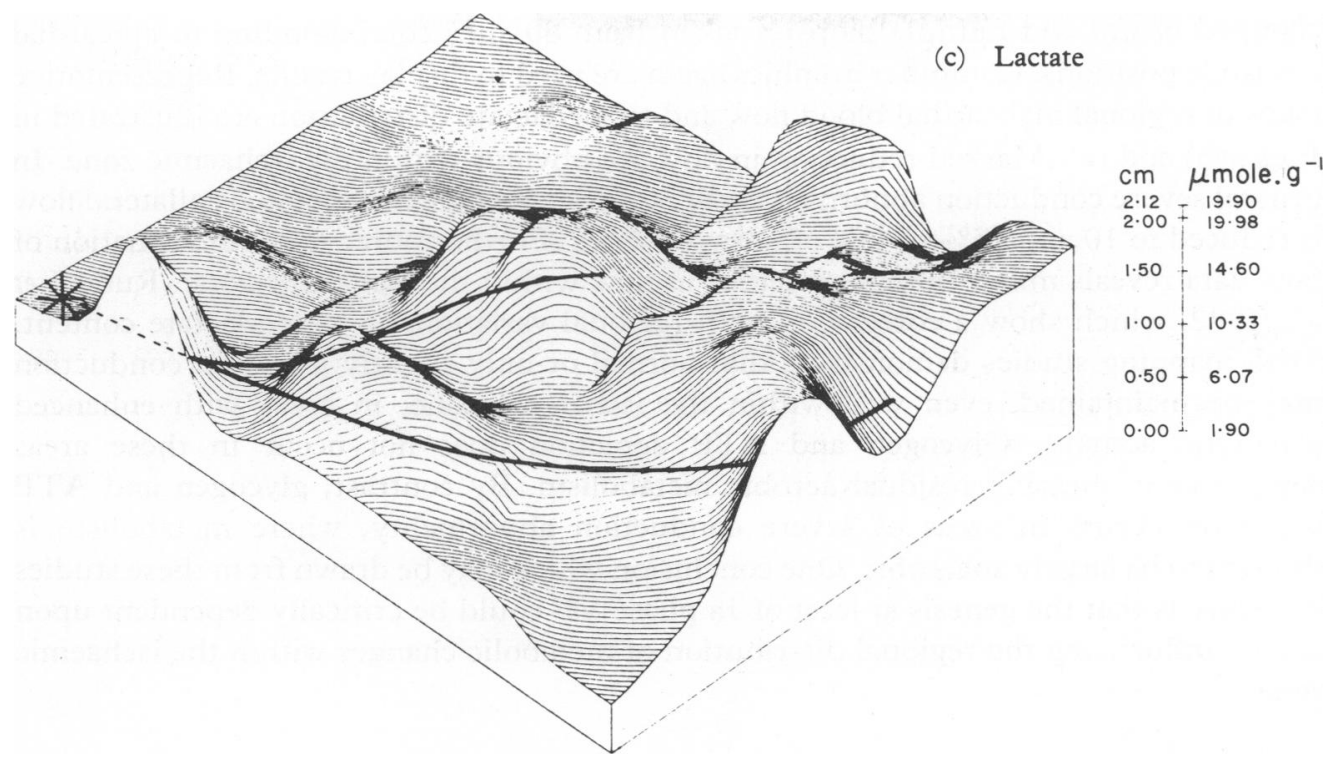

Fig. 3 Representative computer graphic plots of (a) epicardial activation delays; (b) regional myocardial blood flow and (c) tissue lactate distribution in the $4 \times 5 \mathrm{~cm}$ area underlying the electrode grid during phase 1a arrhythmias in the dog (see Fig. 2). The position of the left anterior descending coronary artery is shown by the block line. Horizontal axes refer to grid position. Note the heterogeneity of conduction, flow and tissue lactate distribution in the ischaemic area which is thought to predispose to genesis of VF. (Reproduced by permission)

\section{Patterns of metabolism and genesis of VF}

The cause of these spatial inhomogeneities in electrophysiological properties preceding 1a phase VF is currently under active investigation. One possibility is that they result from underlying spatial inhomogeneities in biochemical responses to ischaemia. Alternatively or associated with this, variability in collateral perfusion of the ischaemic zone could be of importance. Critical modulation of local electrophysiological effects could derive from variations in extracellular potassium or proton accumulation in cellular energy production, adrenergic influences or accumulation of toxic metabolites such as fatty acyl carnitines or lysophosphatides (Corr et al., 1982). Of particular interest to us, has been the concept that variations in cellular metabolic status, in particular the extent of residual aerobic ATP production, might be a most important factor in controlling patterns of conduction within the ischaemic zone and so influence re-entry (Russell et al., 1981).

For these reasons, detailed mapping studies have been performed to correlate surface activation patterns [as shown in Fig. 3(a)] with underlying patterns of flow and metabolism both at onset of and preceding la phase VF. Flow is derived using tracer microspheres and tissue metabolism assessed by analysis of tissue glycogen lactate, high-energy phosphates and indices of regional glycolytic activity. The heart is freeze- 
clamped in situ and mutiple biopsies taken from 80 sites corresponding to epicardia $\overline{\bar{p}}$ electrode positions. Computer graphics again are used to display results. Representative maps of regional myocardial blood flow and tissue lactate distribution are illustrated in Figs 3 (b) and (c). Marked reduction in flow are obvious within the ischaemic zone. In? general severe conduction abnormalities are only observed when residual collateral flow is reduced to 10 and $15 \%$ or less of normal values. Furthermore, closer examination of flow data reveals minor inhomogeneities in flow within the ischaemic zone (Russell $e$ 导 al., 1982) which show a correlation with regional variations in tissue lactate content $\frac{\bar{s}}{\sigma^{\circ}}$ Such mapping studies demonstrate that normal or only slightly impaired conduction? may be maintained, even well within the ischaemic zone, in areas with enhanced? glycolytic activity. Glycogen and ATP depletion does not occur in these areas $\vec{\oplus}$ suggestive of ongoing residual aerobic metabolism. By contrast, glycogen and ATP depletion occurs in areas of severe conduction abnormality, where metabolism is thought to be largely anaerobic. One conclusion which may be drawn from these studie $\stackrel{\mathbb{Q}}{\stackrel{\$}{-}}$ therefore is that the genesis at least of 1a phase VF could be critically dependent uponfactors influencing the regional distribution of metabolic changes within the ischaemic zone.

\section{Adrenergic mechanisms and genesis of VF}

Vulnerability to VF during acute ischaemia is not dependent solely on events within the ischaemic myocardium. Psychological 'trigger factors' may often precipitate Furthermore, these seem more important the less severe the ischaemic injury (Lownecte al., 1978). It is known that sympathetic activity may be enhanced and plasma catecholamine levels elevated within a few minutes of onset of symptoms of myocardia infarction. Experimentally the electrical threshold for induction of VF may be reduced strikingly by sympathetic stimulation (Verrier et al., 1974). Conversely chronic cardia氕 denervation has an anti-arrhythmic effect (Ebert et al., 1968). Such observations suggest that vulnerability to VF in a clinical setting may be greatly influenced by stres responses mediated by the autonomic nervous system.

Possible mechanisms of arrhythmogenicity due to catecholamines or sympathetic noradrenaline release within the ischaemic heart are at present unclear. By extra polation from findings in isolated tissue studies direct electrophysiological effect couldB result from enhancement of membrane polarisation, acceleration of conduction or triggered automatic activity. Conversely, however, opposite effects could result from the metabolic sequelae of increased severity of ischaemic injury with accumulation of toxic intracellular metabolites. Thus, during ischaemia, cardiac glycogen depletion may be accelerated, fatty acid uptake enhanced (in association with elevated levels of plasmå free fatty acids) and inappropriate wasteful energy cycling promoted all of which may have electrophysiological effects. In addition, the role of stimulation of cardio $\tilde{w}^{-}$ sympathetic reflexes in initiation of VF is unclear.

Studies in our laboratory have failed to show significant noradrenaline overflow fromf

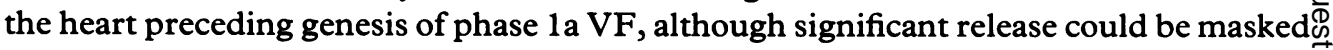
by avid reuptake mechanisms (Riemersma, 1982). Nevertheless, direct left sympathetic stimulation does release noradrenaline within the heart and can increase both the 
severity and heterogeneity of surface conduction abnormalities and induce VF. Effects are maximal during the period of phase la arrhythmias and less so later when the severity of ischaemic injury is greater. Furthermore, these responses may be partially inhibited by beta-blockade, an effect perhaps underlying the prophylactic benefit of beta-blockade against sudden cardiac death.

Many other factors, including modulation of alpha and beta receptor responsiveness and the roles of cyclic AMP and adrenaline and metabolic effects of catecholamines are under current investigation but are beyond the scope of this review.

\section{CONCLUSIONS}

The general conclusion can be drawn from experimental studies, supported by clinical evidence, that VF related cardiac arrest associated with acute myocardial ischaemia or infarction may arise in many ways.

The exact mechanism operating in any one individual will depend upon many factors including duration of ischaemia and 'phase' of arrhythmogenesis, the severity and patterns of ischaemic injury, autonomic effects and degree of systemic metabolic response to ischaemia. Our own research has focussed primarly on elucidating mechanisms of the early la phase of VF. Findings demonstrate the presence of critically timed spatial inhomogeneities of conduction, refractoriness, metabolism and responsiveness to neural stimulation within ischaemic myocardium which could predetermine the generation of re-entrant circuits and hence VF. Mechanisms of later ischaemic VF and reperfusion VF are less clear. Management of cardiac arrest is still largely empirical and continued experimental studies in this field are urgently required to permit a more rational approach to its treatment and prevention. Current evidence would suggest, however, that no single therapeutic approach is likely to be effective as prophylaxis against all forms of cardiac arrest.

\section{REFERENCES}

Allesie M. A., Bonke F. I. M. \& Schopman F. J. G. (1976) Circus movement in rabbit atrial muscle as a mechanism of tachycardia. The role of non-uniform recovery of excitability in the occurrence of undirectional block as studies with multiple micro-electrodes. Circulation Research 37, 168-77.

Armstrong A., Duncan B., Oliver M. F., Julian C. G., Donald K. W., Fulton M., Lutz W. \& Morrison S. L. (1972) Natural history of acute coronary heart attacks. British Heart fournal 34, 67.

Bigger J. J., Dresdale R. J., Heissenbuttel Weld F. M. \& Wit A. L. (1977) Ventricular arrhythmias in ischaemic heart disease: mechanism, prevalence, significance and management. Progress in Cardiovascular Disease 19, 255-300.

Corr P. B. \& Sobel B. E. (1982) Amphiphilic lipid metabolism and ventricular arrhythmias. In Early Arrhythmias resulting from Myocardial Ischaemia. Parrat J. E. (ed). MacMillan.

Cranefield P. F. (1975) The conduction of the cardiac impulse. M. F. Kisco NY Futura Publishing Co.

Ebert P. A., Allgood R. J. \& Sabiston D. C. (1968) The antiarryhythmia effects of cardiac denervation. Annals of Surgery 168, 728-35.

El-Sherif N., Scherlag B. J. \& Lazzara R. (1975) Electrode recordings during malignant ventricular arrhythmias following experimental acute myocardial ischaemia. Circulation 51, 1003-14. 
Janse M. J., van Lapelle F. J. L., Marsin K. H. \& Kleber A. G. (1980) Flow of 'injury' current and patterns oß excitation during early ventricular arryhythmias in acute regional myocardial ischaemia in isolated porcing or canine hearts. Circulation Research 47, 151-65.

Kaplinsky E., Ogawa S., Balke C. W. \& Dreifus L. S. (1979) Two periods of early ventricular arrhythmia ir the canine acute myocardial infarction model. Circulation 60, 393-9.

Kaplinsky E., Ogawa S., Michelsen E. C. \& Dreifus L. S. (1980) Spontaneous and delayed ventricula $\overrightarrow{\overline{\bar{\beta}}}$ arrhythmias after reperfusion of acutely ischaemic myocardium: evidence for multiple mechanisms Circulation 63, 333-40.

Kuller L. (1966) Sudden and unexpected non-traumatic deaths in adults: a review of epidemiological and clinical studies. Fournal of Chronic Diseases 19, 1165.

Lazzara R., El-Sherif N., Hope R. H. \& Scherlag B. J. (1978). Ventricular arrhythmias and electrophysiological consequences of myocardial ischaemia and infarction. Circulation Research 42, 740-9.

Lown B. \& de Silva R. A. (1978) Roles of psychological stress and autonomic nervous system changes ir $\vec{D}$ provocation of ventricular premature complexes. American fournal of Cardiology 41, 979-85.

Riemersma R. A. \& Forfar J. C. (1982) Effects of experimental ischaemia on myocardial catecholamines. Infw Catecholamines in the Non-ischaemic and ischaemic myocardium. Riemersma R. A. \& Oliver M. F. (eds Elsevier Biomedical 139-54.

Russell D. C. \& Oliver M. F. (1978) Ventricular refractoriness during acute myocardial ischaemia and its relationship to ventricular fibrillation. Cardiovascular Research 12, 221-7.

Russell D. C. Smith H. J. \& Oliver M. F. (1979) Transmembrane potential changes and ventriculafo fibrillation during repetitive myocardial ischaemia in the dog. British Heart fournal 42, 88-96.

Russell D. C., Lawrie J. S., Riemersma R. A. \& Oliver M. F. (1981) Metabolic aspects or rhythm disturbances. Acta Medica Scandinavica 230, ii, 71-81.

Russell D. C., Riemersma R. A., Lawrie J. S. \& Oliver M. F. (1982). Patterns of flow and conduction duringD early ventricular arrhythmias following coronary artery occlusion in the dog. Cardiovascular Research $16 \vec{\emptyset}$ 613-23.

Russell D. C. \& Oliver M. F. (1983) Electrophysiological effects of antilipolytic therapy during acgi myocardial ischaemia. Fournal of Molecular Cell Cardiology suppl (in press).

Surawicz B. (1971) Ventricular fibrillation. American fournal of Cardiology 28, 268-87.

Verrier R. L., Thompson P. \& Lown B. (1974) Ventricular vulnerability during sympathetic stimulation: röle of heart rate and blood pressure. Cardiovascular Research i, 602-10.

Wit A. L., Rosen M. R. \& Hoffman B. F. (1974) Electrophysiology and pharmacology of cardiac arrhythmias II. Relationship of normal and abnormal electrical activity of cardiac fibres to the genesis of arrhythmias $\overrightarrow{\overline{0}}$ American Heart fournal 88, 664-70.

Wit A. L. (1982) Electrophysiological mechanisms of ventricular tachycardia caused by myocardial ischemia and infarction in experimental ${ }^{2}$ animals. In Ventricular Tachycardia, Mechanisms and Management ${ }^{1}$. M. FO Kisco NY Futura Publishing Co. 33-9.

Received 14 September 1983; accepted for publication 16 November 1983
This paper was presented at the Scientific Meeting of the Emergency Medicine Research Society, 9-10 September 1983 in York. 